\title{
Adaptación de la técnica SLEDD-F para el tratamiento de un paciente con fracaso renal agudo
}

\author{
Ignacio Fernández de Larrea Varela - M $^{\mathrm{a}}$ Estrella Rebassa Serra - $\mathbf{M}^{\mathrm{a}}$ Mar Castillo Roig
}

Unidad de Diálisis Hospital Son Llatzer. Palma Mallorca

\section{Introducción}

Las alternativas propuestas actualmente para el tratamiento sustitutivo del fracaso renal agudo son la hemodiálisis estándar en pacientes con estabilidad hemodinámica y sin hipercatabolismo, la hemodiafiltración veno-venosa continua en enfermos con inestabilidad hemodinámica e hipercatabólicos y más recientemente la Slow Low Efficient Daily Diálisis (SLEDD) y sus variantes en pacientes estables o inestables hemodinámicamente hipercatabólicos. La elección de una técnica u otra se hace en función de la clínica del paciente y de su tolerancia hemodinámica.

La diálisis mantenida de baja eficiencia SLEDD es un híbrido conceptual y técnico introducido en julio de 1998 en la Universidad de Arkansas que comprende la aplicación de un monitor convencional de hemodiálisis con flujos de dializado y sangre reducidos, para tratamientos, inicialmente nocturnos y de 12 horas de duración. Esta técnica ofrece numerosos beneficios dado que combina las ventajas de la hemodiálisis convencional intermitente con las técnicas dialíticas continuas ${ }^{1}$ : una baja tasa de ultrafiltración

\author{
Correspondencia: \\ Ignacio Fernández de Larrea Varela \\ Unidad de Diálisis Hospital Son Llatzer \\ Ctra Manacor, km 4 \\ 07198 Palma de Mallorca - Islas Baleares \\ ifernandezdelarrea@hsll.es - merebassa@hsll.es \\ mmcastil@hsll.es
}

para optimizar la estabilidad hemodinámica, baja eficiencia en el aclaramiento de solutos para minimizar el desequilibrio de estos y duración mantenida del tratamiento para maximizar la dosis de diálisis². Aún así, la terapia de sustitución renal continua es considerada superior por muchos intensivistas, debido principalmente a la gran cantidad de aclaramiento convectivo conseguido.

Para intentar conseguir una mejor alternativa a las técnicas continuas de UCI, se introducen las variaciones del concepto de SLEDD, principalmente la SLEDD-f, Hemodialfiltración mantenida diaria de baja eficacia, en la que se añade a las ventajas antes mencionadas, la hemodiafiltración, con el consecuente aumento del aclaramiento convectivo. Existe escasa información bibliográfica acerca de de la eficiencia de esta variación de la técnica SLEDD, así que se decidió valorar esa eficiencia usando un nuevo concepto de calculo, el EKRc (equivalente de aclaramiento renal de urea). El EKRjc permite calcular en conjunto la dosis administrada de diálisis durante un periodo de tiempo mayor o menor y aplicarse con diferentes técnicas dialíticas. El EKRjc representa la equivalencia en aclaramiento continuo de urea, en un periodo de tiempo determinado, para aclarar del organismo la cantidad total de urea eliminada mediante diálisis en ese mismo periodo. Su cálculo aunque complejo, no se basa en un modelo iterativo, pudiéndose realizar con una hoja de cálculo Excel. El cálculo se inicia a partir de las determinaciones de BUN pre y post hemodiálisis, además del volumen de distribución de la urea calculado por Fórmula de Watson ${ }^{2}$. En resumen, el equivalente del aclaramiento renal de urea: 
- Representa el aclaramiento continuo normalizado de urea que resulta para una concentración media mantenida en el tiempo que representa una determinada excreción de urea.

- No es un aclaramiento de urea del dializador.

- Es un parámetro de balance de masas que incorpora la ineficiencia de las terapias intermitentes.

- No asigna una dosis mayor de diálisis a los pacientes que inician diálisis con una urea más elevada.

- Integra un cálculo genérico de dosis de diálisis para un periodo de tiempo que incluye tratamientos irregulares con diferentes eficiencias y duraciones, y diferentes generaciones de urea a lo largo del tiempo, aunque no se haya alcanzado el estado de estabilidad de la urea.

EI EKRjc está sustituyendo por tanto al modelo cinético de la urea (KTV) en el cálculo de la dosis en la hemodiálisis de agudos. Con respecto a la dosis objetivo a alcanzar en la diálisis del fracaso renal agudo y el método más apropiado para medir esta dosis, no hallamos bibliografía que determine unas prácticas estándar.

Presentamos el caso clínico de un paciente tratado en nuestra unidad de hemodiálisis de agudos al cual se le aplicó el cálculo EKRjc es decir, el equivalente de aclaramiento renal de urea, para evaluar la efectividad de de la técnica SLEDD-f utilizada, ya que ésta se modificó para adaptarla a las necesidades de la unidad.

\section{Objetivos}

- Calcular la dosis de diálisis en un paciente con fracaso renal agudo hipercatabólico, tratado con la técnica SLEDD-f con reducción del tiempo de las sesiones a 4 horas- 4 1/2 horas, para determinar la eficacia del tratamiento aplicado.

- Identificar ventajas e inconvenientes de la aplicación de la técnica SLEDD-f.

\section{Paciente y método}

Se realizó una revisión bibliográfica acerca de la aplicación de la técnica SLEDD y el cálculo del EKRjc usando las bases de datos: Cuidatge, Cuiden, Pubmed y Google Schoolar.

La experiencia se realizó en la unidad de hemodiálisis del hospital Son LLatzer donde la duración media de las sesiones de HD, HDF-on line es de 4 horas con un margen de \pm 1 hora. La técnica de SLEED-f que realizamos se adaptó, por tanto, a dicho esquema horario. Esto supuso una reducción del tiempo de cada sesión ya que habitualmente el tiempo de dicha técnica es de más de 6 horas $^{3-4}$. El monitor utilizado fue un Fresenius 4008@ con módulo on-line que permite realizar hemodiafiltración on-line.

\section{Resumen de la historia clínica}

Paciente de 51 años de edad, fumador activo y antecedentes de drogadicción, en la actualidad en tratamiento con metadona. Con infección crónica VHC $(+)$, porfiria cutánea y herpes zoster, que acude a consulta por anasarca y hematuria. Con la orientación de síndrome nefrítico (macro/ microhematuria, fracaso renal agudo: $\mathrm{Cr} 7.3 \mathrm{mg} / \mathrm{dl}, \mathrm{HTA}$, proteinuria, edemas y oliguria) ingresa a cargo del servicio de Nefrología. Se asocia a la nefropatía insuficiencia cardiaca derecha por insuficiencia tricuspídea severa (antecedentes de 2 episodios de endocarditis en año 2004). En 2006 ingresa con un diagnóstico de glomerulonefritis membranoproliferativa, con disfunción cardiaca severa e hipertensión pulmonar secundaria. Ante la ausencia de respuesta diurética inicial con perfusión de furosemida EV y la severidad del FRA se procede a colocación catéter tunelizado en yugular derecha para inicio de tratamiento depurador.

Precisó corticoterapia y nutrición parenteral total. El estado hipercatabólico aunque hemodinámicamente estable del paciente y sus características lo hacían subsidiario de la técnica SLEED-f. La pauta que se llevó a cabo fue 6 sesiones semanales durante 3 semanas consecutivas (18 sesiones). El número de horas de diálisis en cada sesión tuvo un intervalo de $4-4,5$ horas, utilizando siempre el mismo filtro de polisulfona Fresenius Fx $80 \circledast$ adecuado a la técnica. La velocidad media de la bomba fue de $250 \mathrm{ccl}$ min, con un intervalo de 200 a $300 \mathrm{cc} / \mathrm{min}$. El flujo de dializante fue de $300 \mathrm{cc} / \mathrm{min}$ en 10 sesiones y de $500 \mathrm{cc} / \mathrm{min}$ en 8 sesiones. El coeficiente de infusión 
fue de media $63 \mathrm{ml} / \mathrm{min}$, con un intervalo de 50 a 84 $\mathrm{ml} / \mathrm{min} \mathrm{El}$ volumen de infusión total fue de media 13.4 litros, con un intervalo de 12.0 a 20.0 litros. Estos valores se obtuvieron calculando la media aritmética.

Se pautó una reducción de $0,5 \mathrm{Kg}$ del peso teórico en cada sesión. Se inició con HBPM a dosis bajas siendo necesario aumentar a HBPM a dosis altas en las dos últimas sesiones por indicios de coagulación de la línea venosa. La concentración de $\mathrm{Ca}, \mathrm{K}, \mathrm{P}$ del baño fue revisada y modificada diariamente en función de los valores analíticos. Para los cálculos de dosis de diálisis se tomaron muestras de sangre antes y después de cada sesión (pre y post hemodiálisis).

El método de cálculo para medir la dosis de diálisis administrada fue el $\mathrm{Kt} / \mathrm{v}$, modelo cinético formal, que representa el aclaramiento fraccional de urea por tratamiento de diálisis ${ }^{3}$. Además, en nuestro caso, y al tratarse de un paciente con fracaso renal agudo, se calculó la dosis de diálisis administrada mediante la fórmula EKRc (equivalente de aclaramiento renal de urea), según describen Casino y $\mathrm{Marshall}^{3}$, que representa el aclaramiento continuo normalizado de urea que resulta para una concentración media mantenida en el tiempo que representa una determinada excreción de urea. Se aplicó hoja de cálculo Excel con formulas preestablecidas.

\section{Resultados}

Los valores de urea pre y post de cada sesión se describen en la tabla 1 . En relación a los datos obtenidos, la urea media antes de la diálisis fue de 111,67 $\mathrm{mg} / \mathrm{dl}$., la urea media obtenida posthemodialisis fue de $45,35 \mathrm{mg} / \mathrm{dl}$., siendo la tasa de aclaramiento de urea del $59.53 \%$.

El cálculo del KTV cinético formal por sesión se representa en la tabla 2. El resultado del cálculo del KTV monocompartimental y bicompartimental de la primera semana fue de 6.258 y de 5.489 respectivamente. En la segunda semana el KTV monocompartimental fue de 6.75 y el bicompartimental de 5.893; y en la tercera semana el KTV monocompartimental fue de 6.67 y el bicompartimental de 5.91 .

En la figura 1 se representa el aclaramiento continuo normalizado de urea de nuestro paciente durante el estudio, siendo el EKRjc sumatorio en la última sesión de 26,91 ml/min. Para llegar a este dato se aplicó la hoja de calculo Excel con la fórmula del EKRjc, que no se incluye aquí debido a su extensión.
En la figura 2 se exponen la evolución del comportamiento de los iones $\mathrm{K}$ y $\mathrm{P}$ durante las sesiones de hemodiálisis, tanto en la determinación analítica pre tratamiento como post-tratamiento.

\begin{tabular}{lcc} 
& Urea prehemodiálisis & Urea posthemodiálisis \\
Sesión 1 & $167 \mathrm{mg} / \mathrm{dl}$ & $79 \mathrm{mg} / \mathrm{dl}$ \\
Sesión 2 & $164 \mathrm{mg} / \mathrm{dl}$ & $72 \mathrm{mg} / \mathrm{dl}$ \\
Sesión 3 & $126 \mathrm{mg} / \mathrm{dl}$ & $47 \mathrm{mg} / \mathrm{dl}$ \\
Sesión 4 & $89 \mathrm{mg} / \mathrm{dl}$ & $36 \mathrm{mg} / \mathrm{dl}$ \\
Sesión 5 & $78 \mathrm{mg} / \mathrm{dl}$ & $31 \mathrm{mg} / \mathrm{dl}$ \\
Sesión 6 & $79 \mathrm{mg} / \mathrm{dl}$ & $34 \mathrm{mg} / \mathrm{dl}$ \\
Sesión 7 & $87 \mathrm{mg} / \mathrm{dl}$ & $39 \mathrm{mg} / \mathrm{dl}$ \\
Sesión 8 & $135 \mathrm{mg} / \mathrm{dl}$ & $50 \mathrm{mg} / \mathrm{dl}$ \\
Sesión 9 & $106 \mathrm{mg} / \mathrm{dl}$ & $39 \mathrm{mg} / \mathrm{dl}$ \\
Sesión 10 & $86 \mathrm{mg} / \mathrm{dl}$ & $36 \mathrm{mg} / \mathrm{dl}$ \\
Sesión 11 & $93 \mathrm{mg} / \mathrm{dl}$ & $34 \mathrm{mg} / \mathrm{dl}$ \\
Sesión 12 & $96 \mathrm{mg} / \mathrm{dl}$ & $38 \mathrm{mg} / \mathrm{dl}$ \\
Sesión 13 & $98 \mathrm{mg} / \mathrm{dl}$ & $42 \mathrm{mg} / \mathrm{dl}$ \\
Sesión 14 & $164 \mathrm{mg} / \mathrm{dl}$ & $58 \mathrm{mg} / \mathrm{dl}$ \\
Sesión 15 & $115 \mathrm{mg} / \mathrm{dl}$ & $47 \mathrm{mg} / \mathrm{dl}$ \\
Sesión 16 & $107 \mathrm{mg} / \mathrm{dl}$ & $40 \mathrm{mg} / \mathrm{dl}$ \\
Sesión 17 & $108 \mathrm{mg} / \mathrm{dl}$ & $44 \mathrm{mg} / \mathrm{dl}$ \\
Sesión 18 & $112 \mathrm{mg} / \mathrm{dl}$ & $44 \mathrm{mg} / \mathrm{dl}$ \\
\hline
\end{tabular}

Tabla 1. Urea pre y posthemodiálisis por sesión

\section{KTV monocompartimental KTV bicompartimental}

\begin{tabular}{lcc} 
Sesión 1 & 0.91 & 0.8 \\
Sesión 2 & 0.988 & 0.87 \\
Sesión 3 & 1.04 & 0.918 \\
Sesión 4 & 1.14 & 0.995 \\
Sesión 5 & 1.14 & 0.995 \\
Sesión 6 & 1.04 & 0.911 \\
Sesión 7 & 1.01 & 0.861 \\
\hline Sesión 8 & 1.21 & 1.06 \\
Sesión 9 & 1.17 & 1.02 \\
\hline Sesión 10 & 1.03 & 0.902 \\
Sesión 11 & 1.18 & 1.03 \\
Sesión 12 & 1.15 & 1.02 \\
Sesión 13 & 1 & 0.883 \\
Sesión 14 & 1.29 & 1.13 \\
Sesión 15 & 1.07 & 0.957 \\
Sesión 16 & 1.15 & 1.01 \\
Sesión 17 & 1.07 & 0.955 \\
Sesión 18 & 1.09 & 0.975 \\
\hline
\end{tabular}

Tabla 2. KTV modelo cinético formal 
[ I. Fernández de Larrea Varela, et al ]

Adaptación de la técnica sledd-f para el tratamiento de un paciente con fracaso renal agudo

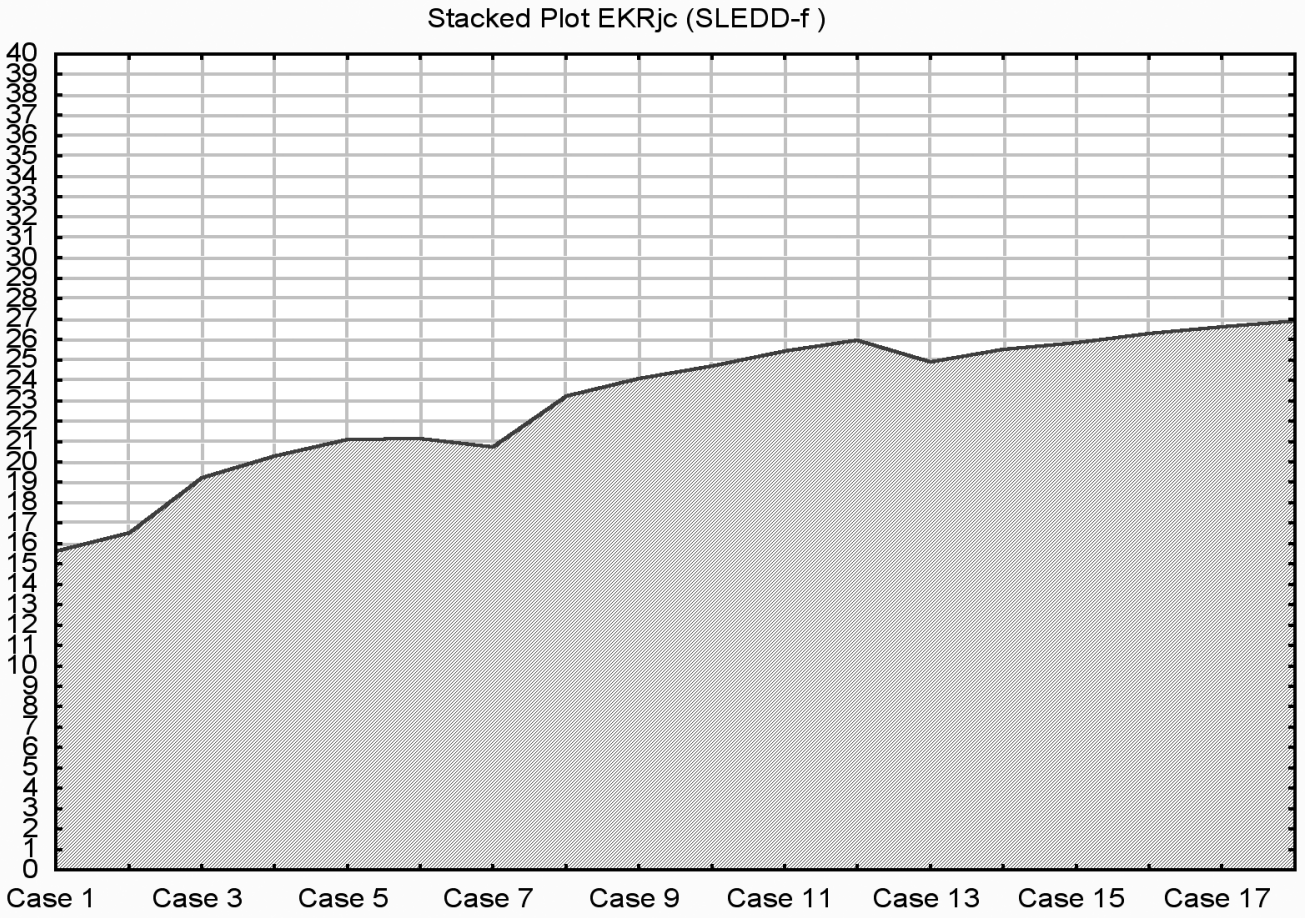

Figural. Equivalente de aclaramiento renal de la urea (EKRjc)

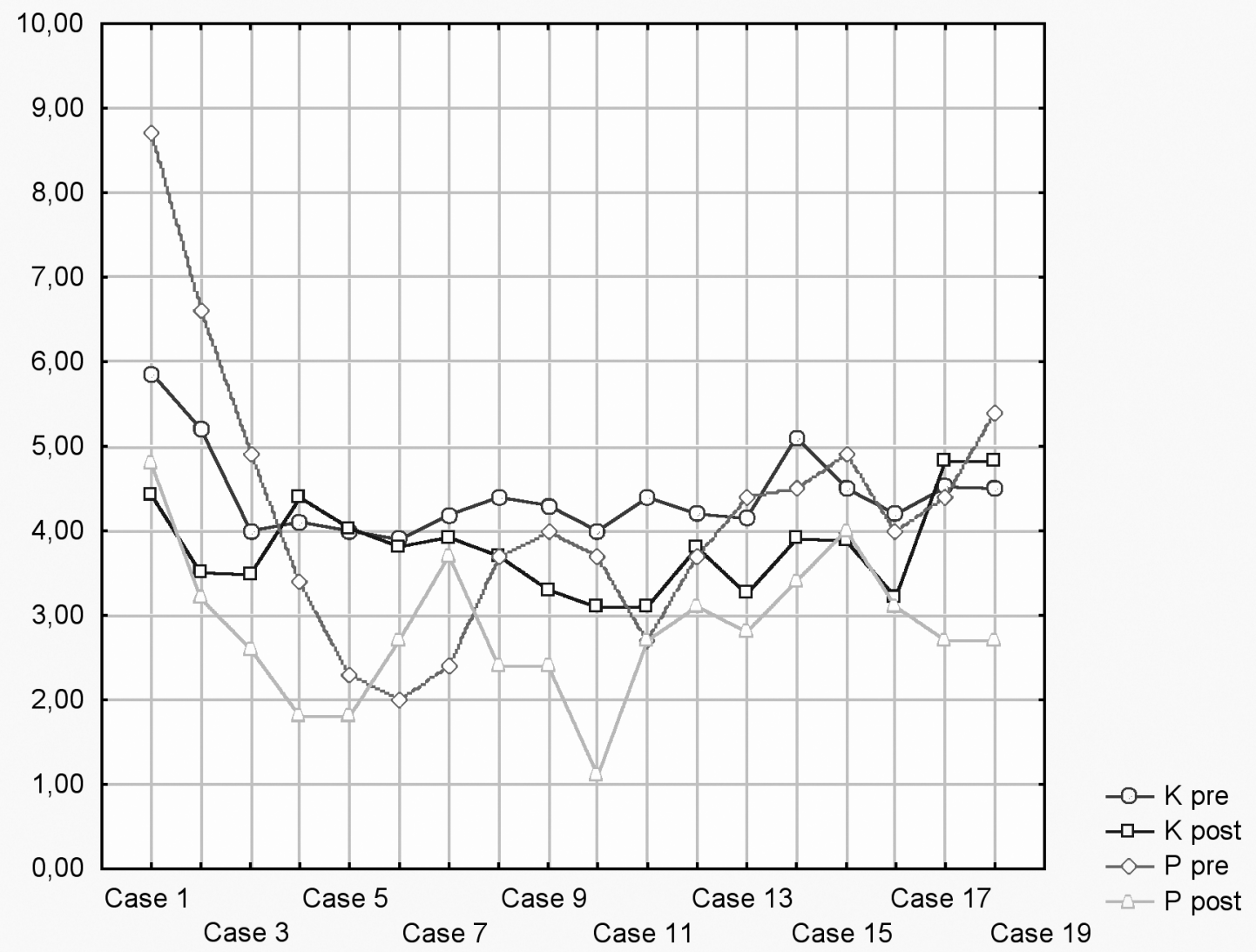

Gráfico 2. Evolución K y P pre y posthemodiálisis 


\section{Discusión y Conclusión}

En nuestro caso hemos observado que la aplicación de la técnica SLEDD-f, con la reducción de tiempo que realizamos, nos ha permitido obtener una dosis de diálisis de 26,91 ml/min; comparado con la bibliografía consultada, según la cual las técnicas continuas para el FRA proporcionarían EKRjc mayores de $30 \mathrm{ml} / \mathrm{min}$ frente un aclaramiento más pobre de menos de $20 \mathrm{ml} / \mathrm{min}$ obtenidos mediante hemodiálisis convencional ${ }^{5}$ podemos decir que el resultado conseguido es muy satisfactorio, ya que se alcanzaron valores que confirman la calidad del tratamiento ofrecido, superiores al de la hemodiálisis convencional.

A nivel de Enfermería los inconvenientes encontrados para la aplicación de la técnica fueron el de un ligero incremento de la carga de trabajo de la unidad de agudos por tratarse de una técnica diaria. Esto mismo, sin embargo, conlleva varias ventajas, ya que el hecho de ser diaria, con ultrafiltraciones más bajas y el alto grado de convección, nos permitió mantener al paciente sin alteraciones de TA ni de FC que provocaran sintomatología alguna y por consiguiente se evitaron las actuaciones intradiálisis por complicaciones hemodinámicas, dato que coincide con publicaciones de hemodiafiltración diaria consultada ${ }^{6-7}$. También la técnica diaria nos permitió reducir el peso seco del paciente en $0,5 \mathrm{Kg}$ por sesión, eliminándose en total $8,6 \mathrm{Kg}$. en 18 días, sin alteración hemodinámica ni presentación de complicaciones relacionadas (calambres, hipotensiones, etc.).

Para el personal de Enfermería no supuso ningún problema adaptarse a la nueva técnica, gracias a la formación recibida y al entrenamiento para la técnica HDF on-line que ya se realiza en nuestra Unidad. Para la aplicación de la SLEDD-f, tal como se hizo en nuestra experiencia, sólo fue preciso modificar los parámetros QB, QD y QI, sin utilizar ningún material adicional ni diferente al habitual en el uso de la HDF on-line.

\section{Agradecimientos}

Agradecemos a todo el personal de Enfermería de la Unidad de Hemodiálisis del hospital Son LLatzer y al jefe de unidad, el Dr. Joan M. Gascó su apoyo y colaboración en la realización de este trabajo, sin la ayuda de los cuales no hubiera sido posible llevarlo a cabo.

\section{Bibliografía}

1. Marshall MR, Golper TA, Sahver MJ Alam MG, Chatoth DK. Urea Kinetics during suatained lowefficiency in critically ill patients requiring renal replacement therapy. Am J Kidney Dis. 2002; 39(3): 556-70.

2. Marshall MR, Golper TA, Sahver MJ, Alam MG, Chatoth DK. Sustained low. Efficiency dialysis for critically ill patients requiring renal placement therapy. Kidney In. 2001; 60(2): 777-85.

3. Casino FG, Marshall MRR, Simple and accurate quantification of dialysis in acute renal failure patients during either urea non-steady state or treatment with irregular or continuous schedules. Nephrology Dialysis Transplantation. 2004; 19:1454-66.

4. Caramelo NJ, Dominguez J. Experiencia sobre SLEDD en cuidados intensivos. Revista Sociedad Española de Enfermería Nefrologica 2005; 8(2):103-107.

5. Clinical Practice guidelines for haemodialysis adequacy. Clinical Practice guidelines and clinical practice recommendations. 2006 Updates. KD0QI. National Kidney Foundation. Disponible en URL:http://kidney.org/professionals/KDOQI/guideline.

6. Berbece AN, Richardson RMA. Sustained low-efficiency dialysis in the UCI: Cost, anticoagulation, and solute removal. Rev. Kidney International 2006; 70,963-968. URL:http://www.kidney-international.org

7. Carreras RM, Cerrillo V, Aicart C, Carratala J, Gil 0 , Muñoz J, Agramunt I. Cambios clínicos y analíticos al pasar de HDF on line convencional a HDF on line diario. En: Libro de comunicaciones presentadas al XXVII Congreso Nacional de La Sociedad Española de Enfermería Nefrológica. Barcelona: HOSPAL; 2002. p. 26-32. 